\title{
A Comparative Analysis of Classification Algorithms in Diabetic Retinopathy Screening
}

\author{
Saboora Mohammadian, Ali Karsaz \\ Electrical Engineering Department \\ Khorasan Institute of Higher Education \\ Mashhad, Iran \\ sa.m.roshan@khorasan.ac.ir, karsaz@khorasan.ac.ir
}

\author{
Yaser M. Roshan \\ Department of Electrical Engineering \\ Point Park University \\ Pittsburgh, PA, US \\ yroshan@pointpark.edu
}

\begin{abstract}
Automated screening of diabetic retinopathy plays an important role in diagnosis of the disease in early stages and preventing blindness in patients with diabetes. Various machine learning approaches have been studied in literature with the purpose of improving the accuracy of the screening methods. In this study a comparative analysis of nine common classification algorithms is performed to select the most applicable approach for the specific problem of screening diabetic retinopathy patients.
\end{abstract}

Keywords-diabetic retinopathy; machine learning; classification

\section{INTRODUCTION}

Diabetic Retinopathy (DR) is an eye disease, which is caused by the damage occurs in the retina due to diabetes. Typically, DR is asymptomatic until it becomes a serious threat for vision; therefore, diagnosing DR at early stages can be crucial to increase the chances of early treatment. By automatic screening, DR can be detected in early stages, while minimizing subjectivity and human errors in the manual approach.

\section{DATASET AND CLASSIFIERS}

\section{A. Diabetic Retinopathy Dataset}

The dataset from UCI Diabetic Retinopathy is used in this study [1]. Features of this dataset have been extracted from the publicly available Messidor database of 1151 fundus images of patients [2]. The input data includes 20 attributes of each of the 1151 images, which includes the images features as discussed in [3], as well as the existence of diabetic retinopathy. For training and testing phases of the classifiers, 30 percent and 70 percent of the available data have been selected randomly as the testing and training set, respectively.

\section{B. Selected Classifiers}

Selected classifiers are, K-Nearest Neighbors, Decision Tree, Naive Bayes, Random Forest, Adaptive Boosting, Quadratic Discriminant Analysis (QDA), Gaussian Process, Support Vector Machine, and Multi-Layer Perceptron (MLP) Neural Network. All of the classifiers are tested and optimized for DR screening. To measure the performance of the classifiers and as a systematic comparison approach, four typical parameters of accuracy, precision, recall, and F1-score are implemented.

\section{RESULTS AND DISCUSSION}

Table I demonstrates the comparison between the best performance values for different classification technique. The Gaussian process classifier demonstrates the best results in terms of classifying the diabetic retinopathy cases, while the SVM with polynomial kernel function (of degree 3) and adaptive boosting approach are also showing acceptable performance.

\section{CONCLUSION}

The study results demonstrate a qualitative comparison between each classifier and its best tuned parameter. Future studies can leverage these results in terms of pre-selecting the classification algorithm and tuning parameters.

TABLE I. Classification Algorithms Performance

\begin{tabular}{|c|c|c|}
\hline Classifier & Optimum Parameter & $\begin{array}{c}\text { Performance Indexes } \\
\text { Accuracy (percision, recall, F1) }\end{array}$ \\
\hline Adaptive Boosting & None & $0.8319(0.8290,0.8305,0.8296)$ \\
\hline Decision Tree & Maximum depth: 2 & $0.7316(0.7573,0.7477,0.7297)$ \\
\hline Naive-Bayes & None & $0.7283(0.7233,0.7176,0.7182)$ \\
\hline Gaussian Process & $\begin{array}{l}\text { Gain: } 4 \\
\sigma: 1\end{array}$ & 0.8707 (0.8796, 0.8799, 0.8707) \\
\hline KNN & Neighbors: 44 & $0.7607(0.8074,0.7802,0.7574)$ \\
\hline QDA & None & $0.6247(0.8709,0.5672,0.4141)$ \\
\hline Random Forest & $\begin{array}{l}\text { Number of trees: } 101 \\
\text { Depth: } 1100\end{array}$ & $0.8028(0.8070,0.8088,0.8026)$ \\
\hline SVM & $\begin{array}{l}\text { Polynomial }(d: 3) \\
\text { Penalty: } 174.5\end{array}$ & $0.8449(0.8651,0.8585,0.8447)$ \\
\hline MLP & $\begin{array}{l}\text { Rectified linear } \\
\text { Hidden layers: } 61 \\
\text { Penalty: } 0.1\end{array}$ & $0.8125(0.8709,0.8190,0.8124)$ \\
\hline
\end{tabular}

\section{REFERENCES}

[1] "UCI machine learning repository: Diabetic Retinopathy Debrecen data set data set," 2014. [Online]. Available:

https://archive.ics.uci.edu/ml/datasets/Diabetic+Retinopathy+Debrecen+ Data+Set\#. Accessed: Nov. 10, 2016.

[2] G. Patry, G. Gauthier, B. Lay, J. Roger, and D. Elie, "ADCIS Download Third party: Messidor database," ADCIS S.A., 2016. [Online]. Available: http://messidor.crihan.fr. Accessed: Nov. 16, 2016.

[3] B. Antal and A. Hajdu, "An ensemble-based system for automatic screening of diabetic retinopathy," Knowledge-Based Systems, vol. 60, pp. 20-27, Apr. 2014. 